\title{
Data Acquisition for Measuring the Wind on Venus from Pioneer Venus
}

\author{
J. R. Smith \\ DSN Data Systems Section \\ R. Ramos \\ Ames Research Center
}

\begin{abstract}
The Pioneer Venus Differential Long Baseline Interferometry experiment was designed to measure the motion in three dimensions of the Pioneer probes during their fall to the surface of Venus, using a combination of Doppler and long baseline ratio interferometric methods. The altitude profiles of wind speed and direction that may be deduced from these data are expected to contribute significantly to the understanding of the dynamics of the Venus atmosphere. The design of the experiment and the equipment and software techniques that were developed specially for this experiment are described.
\end{abstract}

\section{Introduction}

The Pioneer Venus Differential Long Baseline Interferometry (DLBI) experiment was designed to provide precise measurements of the three dimensional velocity profiles for each of the four Pioneer Venus probes during their descent through the Venus atmosphere. For altitudes below about 65 $\mathrm{km}$, the horizontal components of velocity, if averaged over a few seconds, are substantially equal to those of winds. Interpretation of the horizontal velocities at altitudes above about $65 \mathrm{~km}$ and the vertical velocity component at all altitudes requires aerodynamic modeling. The wind velocity information inferred in this way is expected to contribute significantly to the understanding of atmospheric circulation patterns on Venus.

The probe velocity profiles were measured with a combination of direct radial velocity measurements using conventional Doppler velocity measurement technique, and a differential long baseline interferometry technique for the velocity components normal to the line of sight. In a conventional interferometer, the corrupting effects of the transmission media in the vicinity of the spacecraft affect the signal received by both tracking stations almost equally, and therefore largely cancel in the data processing (see Fig. 1). The media in the vicinity of the receiving stations affect the signals independently, and thus the corrupting effects are not reduced by cancellation. In this experiment, the signal from the Bus spacecraft, which remained in ballistic trajectory until after all four probes had impacted the surface of Venus, was used as a reference. Since this Bus signal traversed essentially the same media in the vicinity of the earth stations as did the Probe signals, it was corrupted in essentially the same way. Thus when the interferometric data from the Bus are differenced with the Probe data, the near earth media effects are removed by cancellation. To the extent that the Bus and Probe signals 
are processed by the same ground instrumentation and are corrupted by it in the same way, the effects caused by this instrumentation also tend to cancel in the differencing process. The resulting differenced data are sensitive to the motion of the Probes relative to the Bus spacecraft, and are relatively free of errors introduced by the propagation media and the instrumentation.

Four ground instruments were provided for this experiment. Two used facilities of the Deep Space Network (DSN) stations at Goldstone, CA, and Tidbinbilla, Australia. The other two were located at the Spaceflight Tracking and Data Network (STDN) stations at Santiago, Chile, and Guam (See Fig. 2). The three-dimensional velocity measurements required the use of three stations. The fourth station provided a degree of redundancy which improves the confidence in the resulting data and would have permitted the experiment to be completed should one of the stations have failed to function properly. Each of the instruments made use of the existing antennas and receiver preamplifiers at the stations (see Fig. 3). The DSN stations have $64-\mathrm{m}$ diameter antennas and are equipped with cryogenically cooled maser preamplifiers exhibiting an effective system noise temperature of about $24 \mathrm{~K}$; the STDN stations have 9-m diameter antennas and parametric preamplifiers which provide an effective system temperature of about $100 \mathrm{~K}$. All reference frequency signals and all timing signals were synthesized from a single frequency standard at each station; an atomic hydrogen maser standard was used at each DSN station, and a cesium beam frequency standard was used at each STDN station. The receiver at each station was designed to provide a passband from 2291 to $2293 \mathrm{MHz}$, which was wide enough to include the signals from all four probes and the Bus simultaneously. Additionally, two lowamplitude calibration signals were injected into the receiver, one near each edge of the passband, to permit monitoring of the group delay, the phase delay, and gain of the receiving system. The entire $2-\mathrm{MHz}$ wide passband containing all seven signals was converted by the receiver to a "video" band from 0 to $2 \mathrm{MHz}$ which was then sampled at $0.24-\mu$ s intervals (slightly above the Nyquist rate) and digitized with $3 \mathrm{~b} / \mathrm{sample}$. The resulting $12.5 \mathrm{Mb} / \mathrm{s}$ data stream was then recorded, along with time derived from the local atomic clock, on magnetic tape using a wide-band instrumentation recorder modified for digital recording. A READ-after-WRITE playback capability was then used to provide the data to a fast-Fourier transform spectrum analyzer for verification of overall recording system performance. The signal-to-noise ratio (SNR) that existed at the DSN stations made the unambiguous detection of the Probe signals possible with the spectrum analyzers, but the smaller antenna and higher noise figure of the STDN stations made detection marginal at best, so those stations were operated largely without the benefit of real-time performance verification.

\section{Data Acquisition}

The data tracking phase of the Multiprobe mission, which extended from carrier turn-on of the first Probe until the Day Probe ceased transmitting from the Venusian surface occupied about $2.5 \mathrm{~h}$ during which nearly $10^{12}$ bits of data were recorded. While these data occupied only a modest number of rolls of instrumentation tape, they were not in a form which would have permitted the required data processing. Had this amount of data simply been copied to computer tapes, several thousand reels would have been required. To reduce this amount of data to a manageable level, the wide-band recordings were transported to the Jet Propulsion Laboratory (JPL) where the data were digitally filtered to extract an approximately $1-\mathrm{kHz}$-wide spectral window about each of the seven (5 spacecraft and 2 calibration) signals present in the original $2 \cdot \mathrm{MHz}$ recorded bandwidth. Conventional tracking data were acquired by the two DSN stations during the encounter, and this was used to obtain an a posteriori estimate of the frequency profile of each spacecraft as observed from each station. Figure 4 illustrates a typical Doppler profile. During the digital filtering process at JPL, the spacecraft signals were multiplied by the estimated frequency to remove the major portion of the Doppler shift and the result filtered to the $1 \cdot \mathrm{kHz}$ bandwidth. This bandwidth reduction process reduced the amount of data to about $4 \times 10^{9}$ bits which, although large, was manageable. The computer tapes, containing the bandwidth-reduced data, were shipped to the Massachusetts Institute of Technology (M.I.T.) where the experimenter and his associates are continuing the data processing.

\section{Data Processing}

The data processing is a multipass process in which the phase history of each signal at each station is determined. These histories are then differenced twice. First the phase for the same spacecraft is differenced for two receiving stations, then these differences for two spacecraft (a Probe and the Bus) are differenced to produce the desired information on the vector component of the velocity of the Probe relative to the Bus. This vector component lies in the plane defined by the two stations and the Probe and is normal to the average line of sight between the stations and the Probe. Further processing, involving knowledge of the Bus trajectory relative to the planet and knowledge of the Probe position relative to Venus, is necessary to define the direction of the local vertical at the Probe. Further discussion here will be concerned only with the determination of the phase history of the Probe signals as observed at the stations.

The signals recorded at the DSN stations exhibit a sufficiently high SNR that they may be phase tracked without difficulty. This is done using a process that is essentially a 
digital model of the standard analog phase-locked loop. For the signals recorded at the STDN stations, however, the problem is more difficult because the signals are undetectable with less than a few seconds averaging time, and the rate of change of frequency remaining in the signals after the first step of processing at JPL is too high to permit tracking the signals with a sufficiently narrow loop. To alleviate this problem, the frequency profile observed at a DSN station is adjusted by the expected Doppler difference between that station and a STDN station and then used to remove most of the remaining frequency variation. The resulting data are then digitally filtered to a $20-\mathrm{Hz}$ bandwidth in which the signal can be tracked with a loop utilizing a 10-s time constant.

The SNR in this loop is approximately 10 , but the phase error is as large as 0.5 radian and there are phase slips and periods of loss of lock caused by the rapid (relative to the averaging time) fluctuations of signal frequency. The frequency profile thus determined is then removed from the data, and the process is iterated several times. The resulting phase profile is the best available estimate of the phase profile of the signal as observed at the STDN station, but may still contain errors due to cycle slips or loss of lock in the phase-locked loop used. As a check on the quality of the data, the process is repeated, starting with the measured frequency profile from the other DSN station. The results typically agree to within the noise in the DSN data (6 degrees peak to peak) which is much less than the noise in the STDN data. This is, of course, a necessary but not sufficient condition to guarantee correct phase tracking. To gain additional confidence, the data are reprocessed using different integration times for the tracking loop. When a 6-s averaging time is used, the data are degraded by the remaining thermal noise, while for an 18-s averaging time, the actual frequency changes due to the varying Venus winds degrade the quality of the phase tracking. As a final check, the redundancy provided by the fourth observing station is used; the velocity profiles, as determined using data from the two DSN stations and one STDN station, are compared with the profiles using the two DSN stations and the other STDN station. Since the errors will occur largely as a result of attempting to phase track the weak STDN data, this last comparison provides considerable confidence that the profiles are correct to the level of the agreement observed. It is believed that the estimates of the velocity of the probes, relative to Venus and including all significant error sources, will be accurate to $0.3 \mathrm{~m} / \mathrm{s}$ for all components when averaged over 100-s intervals.

\section{Instrumentation}

Much of the equipment used to support the DLBI experiment was standard tracking station equipment. This standard equipment includes the antenna and antenna-pointing equipment, the low-noise receiver preamplifiers, and the frequency standards and clocks. The receivers were a special wide-bandwidth single-sideband design for which phase stability was a major design consideration. A block diagram of the receiving system used at the DSN stations is shown in Fig. 5. Performance requirements for the system are presented in Table I. The receivers used in the STDN stations were similar except that parametric preamplifiers were used rather than maser preamplifiers. The calibration tones were generated by a tunnel diode pulse generator driven by a phase stable $5.4-\mathrm{MHz}$ square wave. The pulse train was then gated at a $1.8 \mathrm{MHz}$ rate to produce a 1.8 million pulse per second pulse train. Harmonics of these pulses at $2291.1 \mathrm{MHz}$ and $2292.9 \mathrm{MHz}$ were then injected into the receiver. At the DSN station, the calibration signals were injected after the distribution amplifier that delivers the signals to the receivers used for telemetry so as to avoid interference with the telemetry recovery process. Provision was made, however, for injecting the calibration signals ahead of the maser preamplifier for test purposes. Since the STDN stations were dedicated to this experiment and were not attempting to recover the telemetry data, the calibration signals were injected ahead of the preamplifiers at those stations. The remainder of the receiver is of conventional design except for the three blocks shown at bottom center of Fig. 5. These blocks provided a local monitor of the phase stability of the receiver that could be used during testing. The amplitude of the calibration tones used during the mission was too low to allow this equipment to function during this time. The mixer and bandpass filter separated the calibration tones present in the receiver output and mixed them together to obtain a signal at the $1.8 \mathrm{MHz}$ tone spacing. The phase meter compared this with a model of the original pulse repetition rate derived from the same source as the original pulses. This comparison provides a good indicator of the phase stability of the receiver.

The output of the receiver, which contained the five spacecraft signals and the two calibration tones all dominated by the thermal noise present in the $2-\mathrm{MHz}$ bandwidth, was then sampled at $0.24-\mu$ s intervals and quantized to 3 bits. The quantizer was designed with optimally spaced quantization levels to minimize the degradation in SNR caused by the quantizing. The quantized digital data, together with timing and error detection information, were then recorded on an instrumentation tape recorder which was specially modified for digital recording. The data were distributed across twenty tracks on the tape and recorded at a bit density of $11000 \mathrm{~b} / \mathrm{cm}$ $(28000 \mathrm{~b} / \mathrm{in})$. This permitted the recording of the $12.5 \mathrm{Mb} / \mathrm{s}$ data at a tape speed of $11.8 \mathrm{~cm} / \mathrm{s}(30 \mathrm{in} / \mathrm{s})$ which provided about $80 \mathrm{~min}$ of recording per reel of tape.

As discussed above, the wide-band tapes recorded at the four tracking stations were processed at JPL to produce 
narrow-bandwidth records. Each tape contained the signal from one spacecraft or one calibration signal. The processing was entirely digital and was performed using special purpose hardware in conjunction with a general purpose computer. A conceptual block diagram of the process is given in Fig. 6. The a posteriori estimate of the frequency profile for one signal was used by the control computer to control the frequency of the oscillator. The time base for this process was provided by the wide-band data samples, and the oscillator actually produced a sequence of numbers representing the oscillator output. Because this process was fully quantized in both time and amplitude, the phase history of the oscillator was exactly known. The oscillator output was multiplied by the signal samples in the mixer and the result summed in the filter. The running sum was sampled periodically and stored on the output tape. The quadrature channel was provided to allow separation of signals whose frequencies were above the oscillator frequency from those whose frequencies were below. This process was repeated for each of the seven input signals on each wide-band tape from each of the four stations. The resulting narrow-band data, in standard computer tape format, was then sent to M.I.T. for further processing by the experimenter.

Preliminary results reported by the experimenter (Refs. 1 and 2) show that the recording equipment at all four stations functioned perfectly, and that the velocities of the winds on Venus can be estimated from the data.

\begin{abstract}
Note
This article originally appeared in IEEE Transactions on Geoscience and Remote Sensing, Vol. GE-18, No. 1, January 1980.
\end{abstract}

\title{
Acknowledgments
}

The conduct of the DLBI experiment required the dedicated efforts of a great many people for the design, implementation, and performance verification of the many pieces of equipment and computer programs that were required. Many additional people were involved in testing and operating the equipment. We can thank only a few of these. The crews of the four tracking stations performed flawlessly in their execution of these intricate observations; D. W. Johnston of JPL and G. Kronmiller, J. McKenzie, and P. Mitchell of the Goddard Space Flight Center provided the training and operations support for the DSN and STDN; P. Liebrecht of GSFC assisted in overall coordination for the STDN; H. Donnelly and associates (JPL) implemented the receiver and calibrated systems; K. Kimball and associates (JPL) provided the wide-bandwidth recorders; R. Tappan (JPL) implemented the bandwidth reduction assembly; R. Speer and associates (Bendix Field Engineering Corp.) operated the bandwidth reduction assembly. I. I. Shapiro of M.I.T. originally suggested the DLBI experiment in 1971 and G. H. Pettengill, Chairman of the Pioneer Venus Radio Science Team led the effort in 1972 to 1974 to include the experiment in the Multiprobe mission plan. C. C. Counselman, III, of M.I.T. is the experimenter. His exceptional level of interest and enthusiasm contributed enormously to the success of the implementation and operations activities. S. A. Gourevitch, R. W. King, G. B. Loriot, and R. G. Prinn, all of M.I.T., provided the data processing software and are providing the data analysis. R. B. Miller (JPL) is the DSN Tracking and Data Systems Manager for the Pioneer Venus Project; L. Colin (Ames Research Center) is the Project Scientist and C. Hall (ARC) is the Pioneer Venus Project Manager. 


\section{References}

1. C. C. Counselman, III, S. A. Gourevitch, R. W. King, G. H. Pettengill, R. G. Prinn, I. I. Shapiro, R. B. Miller, J. R. Smith, R. Ramos, and P. Liebrecht, "Wind velocities on Venus: Vector determination by radio interferometry," Science, Vol. 203, p. 805, 1979.

2. C. C. Counselman, III, S. A. Gourevitch, R. W. King, G. B. Loriot, and R. G. Prinn, "Venus winds are zonal and retrograde below the clouds," Science, Vol. 205, p. 85, 1979.

Table 1. Performance specifications on the DLBI recelvers

\begin{tabular}{|c|c|}
\hline Parameter & Requirement \\
\hline $\begin{array}{l}\text { Input signal frequency range } \\
\text { for probe and calibration } \\
\text { signals }\end{array}$ & 2291.1 to $2292.9 \mathrm{MHz}$ \\
\hline \multicolumn{2}{|l|}{ Phase calibrator } \\
\hline $\begin{array}{l}\text { Phase stability } \\
\text { (over four hours) }\end{array}$ & $\begin{array}{l}\leqslant 1 \text { deg phase variation between } \\
\text { calibration signals }\end{array}$ \\
\hline Signal level & $\begin{array}{l}\geqslant 94 \mathrm{dBm} \text { into receiver front end via } \\
\text { coupler }\end{array}$ \\
\hline Frequencies & $\begin{array}{l}2291.1 \text { to } 2291.15 \mathrm{MHz} \text { lower calibra- } \\
\text { tion signal; } 2292.9 \text { to } 2292.95 \mathrm{MHz} \\
\text { upper calibration signal }\end{array}$ \\
\hline \multicolumn{2}{|l|}{$\begin{array}{l}\text { Receiver composite output } \\
\text { signal }\end{array}$} \\
\hline Frequency range & 0.1 to $1.9 \mathrm{MHz}$ \\
\hline Phase ripple & 2 deg peak-to-peak \\
\hline Phase linearity & $\begin{array}{l}\text { 9-pole Tchebycheff response. Phase } \\
\text { equalized to within } 10 \% \text { of linear }\end{array}$ \\
\hline Amplitude response & $\geqslant 10 \mathrm{~dB}$ down at $2.26 \mathrm{MHz}$ \\
\hline
\end{tabular}




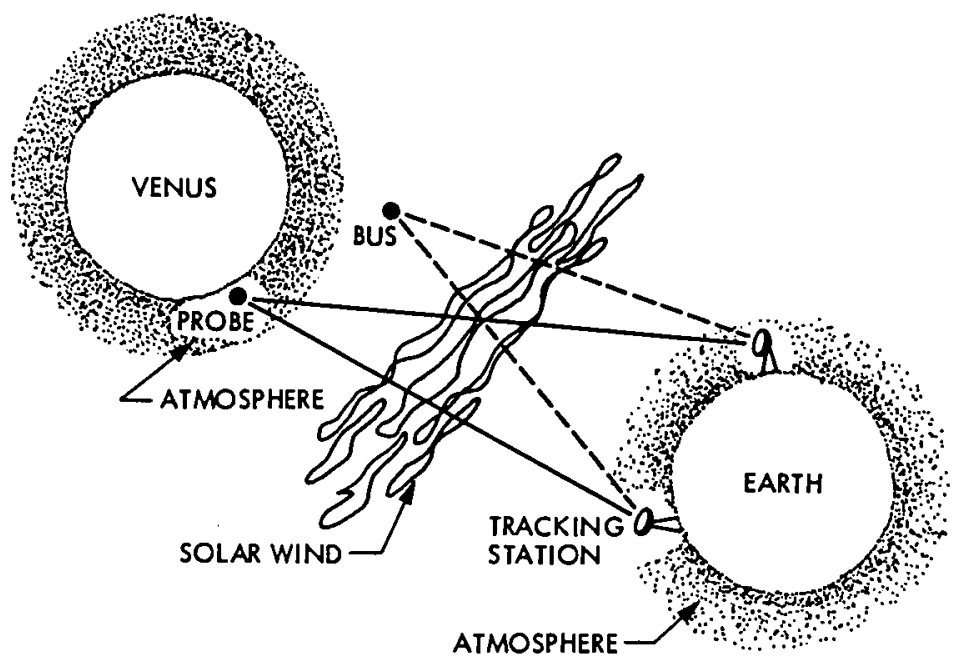

Fig. 1. Geometry of the DLBI observations, with the atmospheric and the interplanetary propagation media indicated. Because the propagation paths are so nearly parallel, and because the DLBI observable is differenced symmetrically with respect to both the transmitting spacecraft and the receiving ground stations, the effects on the observable of both Venus' and the earth's atmospheres cancel to a high degree. The effects of the interplanetary plasma do not cancel so exactly

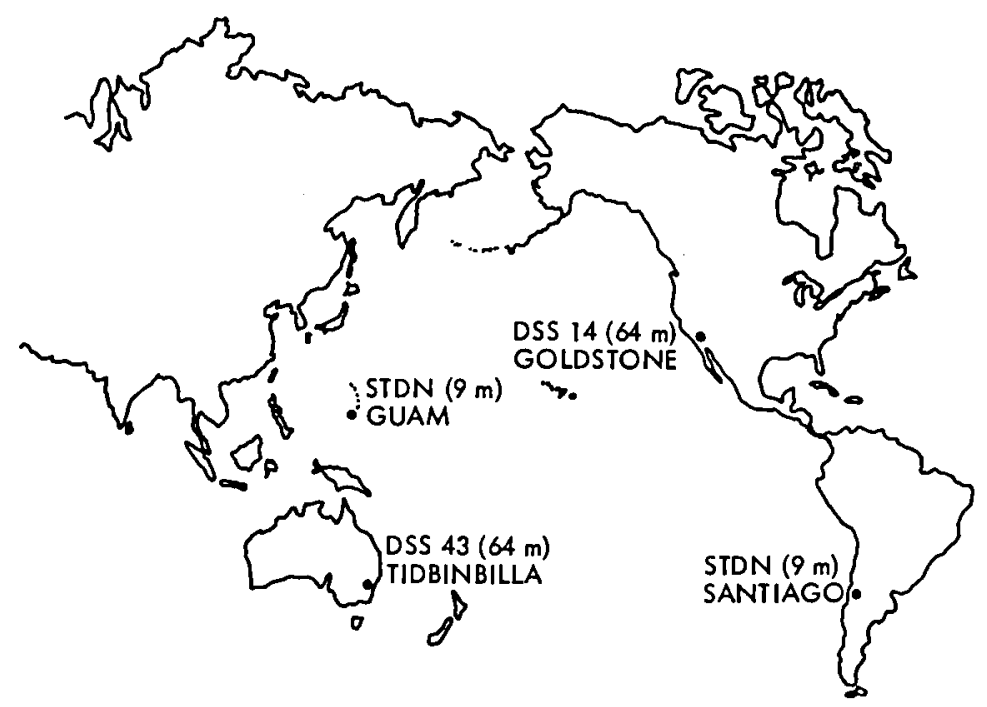

Fig. 2. DSN and STDN station locations, DLBI wind measurement experiment 


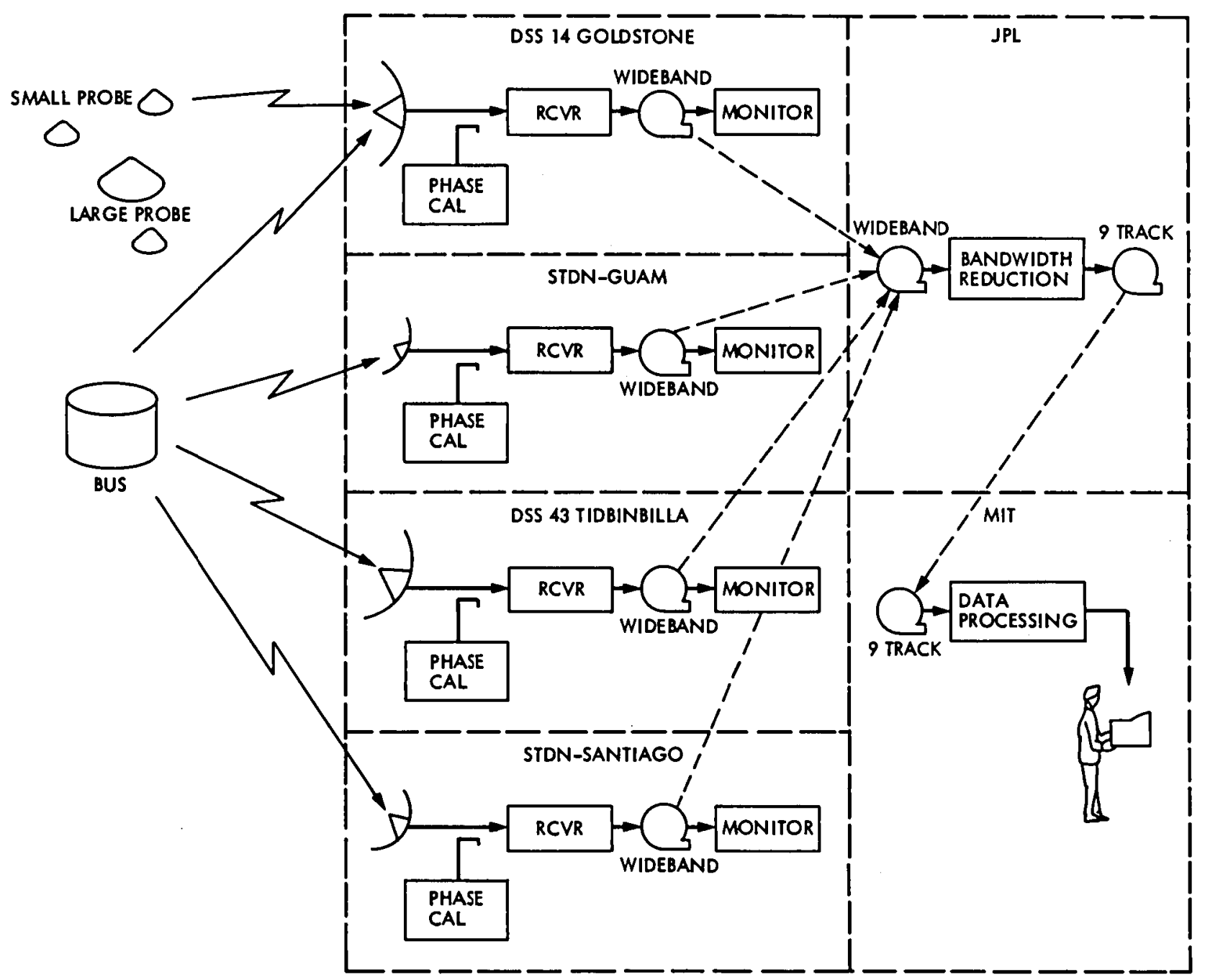

Fig. 3. The DLBI experiment involved the simultaneous reception and recording of the signals from the four Ploneer Probes and the Bus spacecraft, and subsequent processing of the recorded data at JPL and M.I.T. 


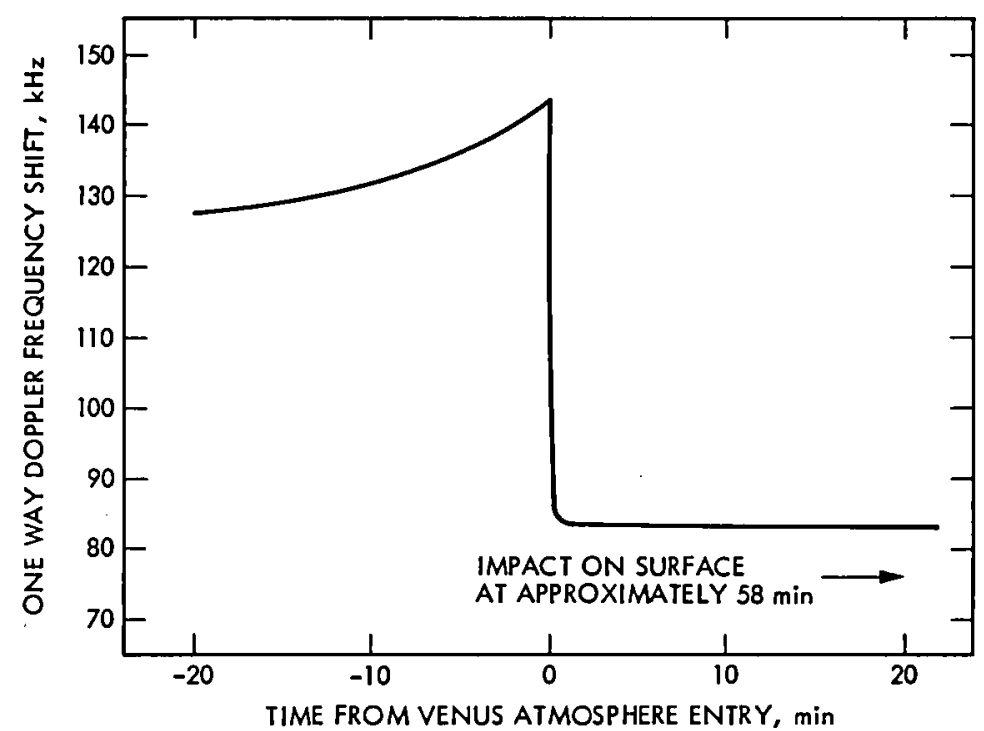

Fig. 4. The Pioneer Venus Probes fell with increasing speed as they approached Venus. Upon reaching the top of the Venus atmosphere, they were quickly slowed by atmospheric friction and then fell through the atmosphere at terminal velocity. This velocity profile results in a Doppler shift in the Probe's S-band radio carrier frequency. A typical frequency profile for the Ploneer Venus Probes is illustrated 


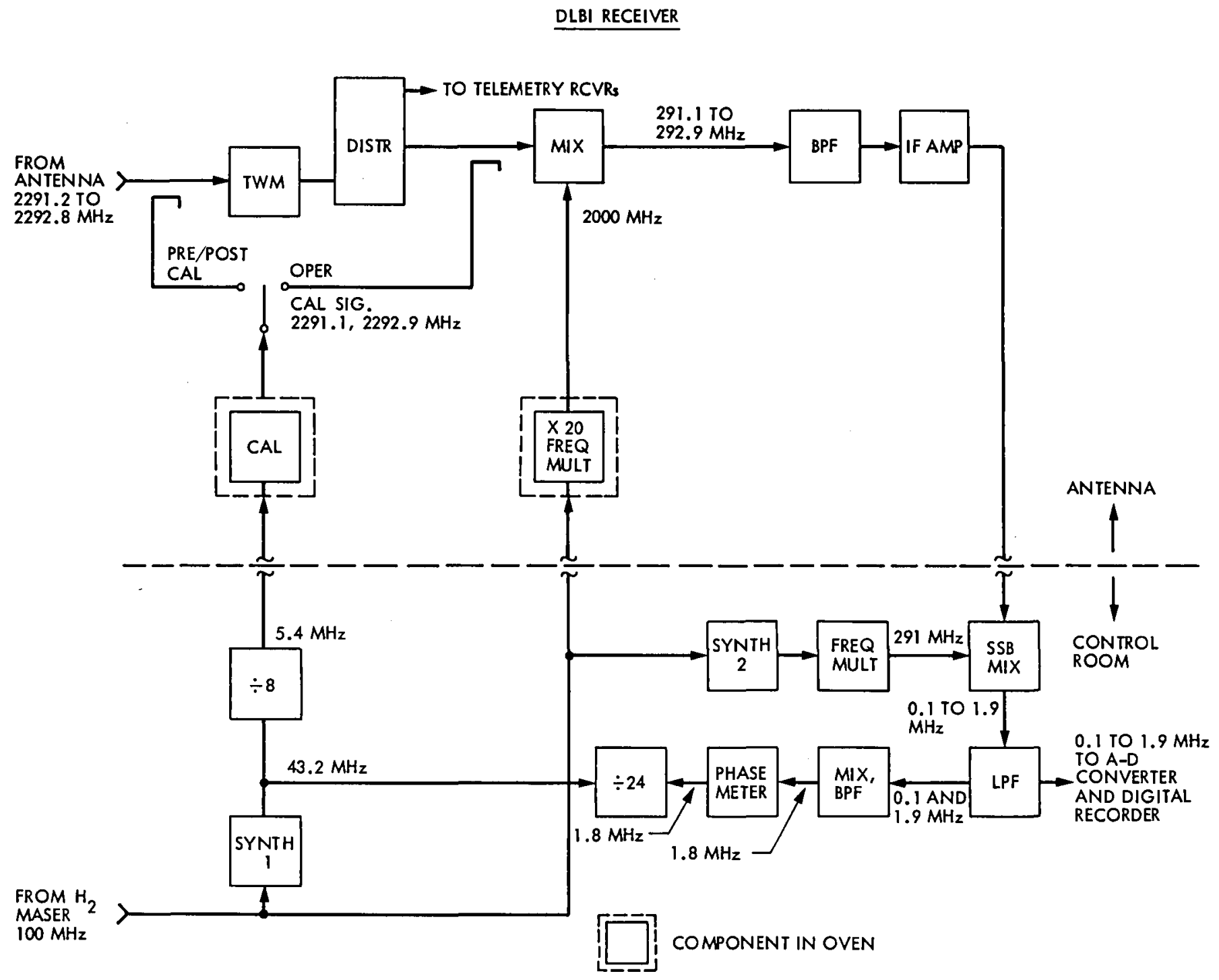

Fig. 5. Simplified block diagram of the receiving system used in the DLBI experiment 


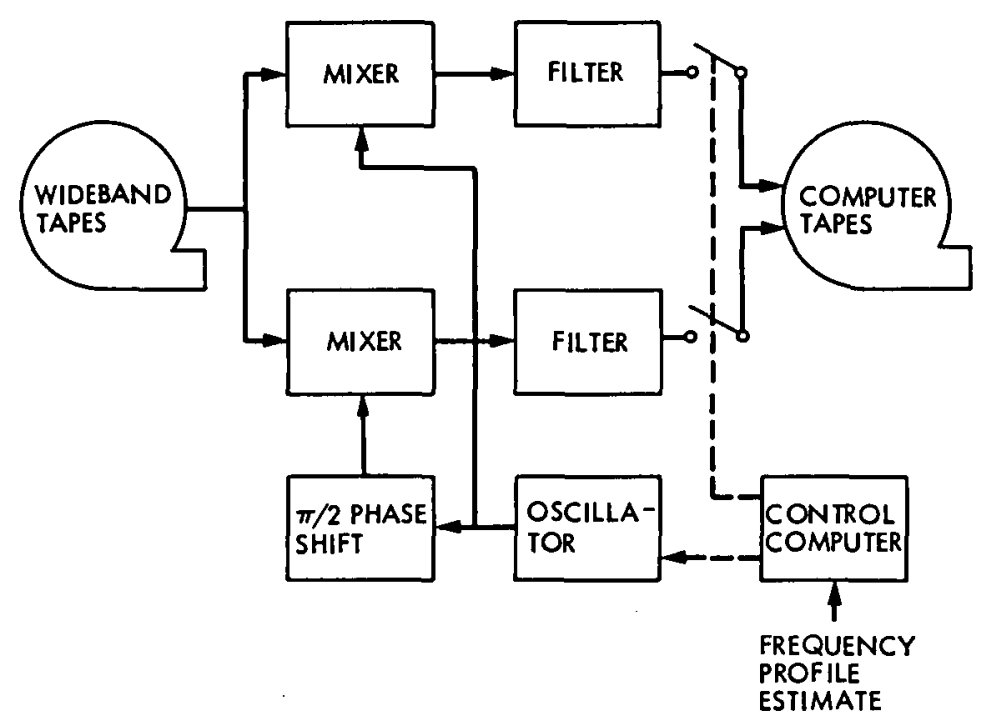

Fig. 6. Simplified functional block diagram of the bandwidth reduction system provided for the DLBI experiment at JPL 